\title{
Annex - Scope of Clean Technologies
}

The list below is generated from the UNEP DTU partnership database on Technology Needs Assessment (TNA). It shows technologies prioritised by 29 developing countries undergoing the tna process between 2011 and 2013.

Sector/ Technology class Within class varieties
sub-sector

Solar energy Solar power

Solar heating/

drying

Wind

energy

Hydro

power

Biofuels Biogas
Solar homes, PV systems, Lanterns, DC motors for grid connected PVs

Solar dryers and water heaters

Wind turbines, Standalone and gridconnected systems, On-shore plants, Systems for water/space heating Micro, small, and large hydro power plants, Pumps to supply water to reservoirs

Biogas for cooking, heating and electricity, Anaerobic digester, Manure digestion, Compact digester for urban households, System lagoons

Biomass
gasification

Biodiesel

Second generation biofuels
Biomass gasifier, Electricity and heat cogeneration plants, Direct combustion technology for electricity generation, CoInternal combustion, Generating biodiesel from jatropha, soy bean and sunflower, Blending with fossil fuel, Fuel replacement (with conventional diesel)

Biofuel from sugarcane, Ethanol gel fuel and fuel-based lanterns, Bioethanol from maize, sugar and starch based crops, Ethanol production from agricultural residues firing of biomass with coal, Waste to energy 


\begin{tabular}{|c|c|c|}
\hline $\begin{array}{l}\text { Sector/ } \\
\text { sub-sector }\end{array}$ & Technology class & Within class varieties \\
\hline \multirow{4}{*}{$\begin{array}{l}\text { Other } \\
\text { energy } \\
\text { sources }\end{array}$} & Nuclear & $\begin{array}{l}\text { Low power nuclear C HP, Long-term large } \\
\text { scale units, Nuclear units of small capacity }\end{array}$ \\
\hline & Coal & $\begin{array}{l}\text { High efficiency boilers, Pulverized coal } \\
\text { combustion (single and double unit), } \\
\text { Integrated coal gasification, Underground } \\
\text { gasification, Co-firing with biomass, } \\
\text { Integrated gasification combined cycle } \\
\text { (IGCC) units, RDF production and pyrolysis } \\
\text { from coal and syngas }\end{array}$ \\
\hline & Natural gas & $\begin{array}{l}\text { Natural gas combined cycle, Fuel switch } \\
\text { to natural gas at existing plants, Micro and } \\
\text { mini combined heat and power ( } \mathrm{CHP}) \\
\text { plants }\end{array}$ \\
\hline & Geothermal & $\begin{array}{l}\text { Geothermal hot water, Geothermal power } \\
\text { technology }\end{array}$ \\
\hline \multirow[t]{2}{*}{$\begin{array}{l}\text { Other } \\
\text { energy } \\
\text { technologies }\end{array}$} & $\begin{array}{l}\text { Combined } \\
\text { cycle (CC), and } \\
\text { combined heat } \\
\text { and power ( } \mathrm{CHP})\end{array}$ & $\begin{array}{l}\text { Peat based IGCC, Biogas based co- } \\
\text { generation, Natural gas combined cycle, Co- } \\
\text { generation by single combined cycle, }\end{array}$ \\
\hline & $\begin{array}{l}\text { Energy efficient } \\
\text { transmission } \\
\text { carbon } \\
\text { capture and } \\
\text { sequestration/ } \\
\text { storage }\end{array}$ & $\begin{array}{l}\text { Supply side transmission, Transmission } \\
\text { lines upgrade, Smart grids }\end{array}$ \\
\hline \multirow{5}{*}{$\begin{array}{l}\text { Forestry and } \\
\text { Agriculture }\end{array}$} & Afforestration & Afforestation using machines, scattered tree \\
\hline & $\begin{array}{l}\text { and reforestration } \\
\text { Agroforestry }\end{array}$ & $\begin{array}{l}\text { planting, improving roadside vegetation } \\
\text { Soil moisture retention, Mechanization of } \\
\text { biomass production }\end{array}$ \\
\hline & $\begin{array}{l}\text { Crop } \\
\text { management }\end{array}$ & $\begin{array}{l}\text { Weed control, Change of crops, Intensive } \\
\text { rice cultivation, Wet and dry irrigation }\end{array}$ \\
\hline & $\begin{array}{l}\text { Forest } \\
\text { management }\end{array}$ & $\begin{array}{l}\text { Optimal plantation, Community forest } \\
\text { management, Mangrove restoration }\end{array}$ \\
\hline & $\begin{array}{l}\text { Fertilizer } \\
\text { management }\end{array}$ & $\begin{array}{l}\text { Conservation tillage, Controlled application } \\
\text { of nitrogen fertilizer, Fixators of free } \\
\text { nitrogen, Inhibitors of volatilitization of } \\
\text { urea }\end{array}$ \\
\hline
\end{tabular}




\section{Sector/ Technology class Within class varieties sub-sector}

\begin{tabular}{|c|c|c|}
\hline & $\begin{array}{l}\text { Tillage } \\
\text { management }\end{array}$ & $\begin{array}{l}\text { Replacement of plow, Mini-till and no-till } \\
\text { soil cultivation system }\end{array}$ \\
\hline & $\begin{array}{l}\text { Peat carbon } \\
\text { management }\end{array}$ & $\begin{array}{l}\text { Peat measurement, monitoring and } \\
\text { remapping, Peat water management }\end{array}$ \\
\hline & REDD & Reducing emissions from forest degradation \\
\hline & Storage and & Different storage practices, Decanting, \\
\hline & handling & Filtration \\
\hline & Organic farming & $\begin{array}{l}\text { Mirco dose technique, Use of organic } \\
\text { manure, Use of sludge from wastewater } \\
\text { treatment plants }\end{array}$ \\
\hline & $\begin{array}{l}\text { Efficient charcoal } \\
\text { production }\end{array}$ & Improved kilns \\
\hline & Feeding practices & $\begin{array}{l}\text { Controlled fodder supplements, Nutrition } \\
\text { improvement through fodder processing, } \\
\text { Growth hormones }\end{array}$ \\
\hline & Others & Land management, Agronomy \\
\hline \multirow{5}{*}{$\begin{array}{l}\text { Mining and } \\
\text { Industry }\end{array}$} & End-use & Efficiency in cement industry, Using \\
\hline & efficiency & pozzolans \\
\hline & $\begin{array}{l}\text { Oven and furnace } \\
\text { efficiency }\end{array}$ & $\begin{array}{l}\text { Autogenerating crisol oven, Induction } \\
\text { furnace, Autorecuperative burners, Forced } \\
\text { air furnace }\end{array}$ \\
\hline & $\begin{array}{l}\text { Efficient brick } \\
\text { kilns }\end{array}$ & Retort, Vertical shaft brick kilns \\
\hline & $\begin{array}{l}\text { Coal mine } \\
\text { methane recovery }\end{array}$ & Enhanced peat / coal-bed methane recovery \\
\hline \multirow{3}{*}{$\begin{array}{l}\text { Residential } \\
\text { and } \\
\text { Commercial }\end{array}$} & Efficient lighting & $\begin{array}{l}\text { Compact fluorocent lamps, LED lights, High } \\
\text { efficiency lighting systems }\end{array}$ \\
\hline & Efficient stoves & $\begin{array}{l}\text { Improved stoves (charcoal, firewood, and } \\
\text { biomass) for cooking and heating, Mud } \\
\text { stoves, Radiation stoves, Ethanol and LPG } \\
\text { cook stoves }\end{array}$ \\
\hline & Efficient heating & $\begin{array}{l}\text { High and medium power heat pumps, } \\
\text { Radiant panels, Hydrogen heating systems, } \\
\text { Efficient heat, ventilation, and air- } \\
\text { conditioning (HVAC) systems, Heat metering, } \\
\text { Automatic temperature regulation }\end{array}$ \\
\hline
\end{tabular}




\section{Sector/ Technology class Within class varieties sub-sector}

\begin{tabular}{|c|c|c|}
\hline & Insulation & $\begin{array}{l}\text { Building thermal insulation, Wall } \\
\text { insulation, Energy protective glasses and } \\
\text { pellicles }\end{array}$ \\
\hline & Less energy & Compressed stabilized earth blocks (CSEB), \\
\hline & $\begin{array}{l}\text { intensive } \\
\text { products }\end{array}$ & $\begin{array}{l}\text { Efficient coolers, Improving battery/ } \\
\text { capacitors' power factors, Efficient air- } \\
\text { conditioning, Cans with paper bottoms }\end{array}$ \\
\hline & Building & Energy management systems, Prefabricated \\
\hline & efficiency & houses \\
\hline & $\begin{array}{l}\text { Household end- } \\
\text { use efficiency }\end{array}$ & $\begin{array}{l}\text { Industrial and commercial end-use energy } \\
\text { efficiency, Household end-use energy } \\
\text { efficiency }\end{array}$ \\
\hline & $\begin{array}{l}\text { Carbon footprint } \\
\text { of products }\end{array}$ & Calculating carbon footprints \\
\hline \multirow[t]{9}{*}{ Transport } & $\begin{array}{l}\text { Bus rapid transit } \\
\text { system }\end{array}$ & $\begin{array}{l}\text { Bus rapid transit (BRT), Transport } \\
\text { management system }\end{array}$ \\
\hline & Mass transport & $\begin{array}{l}\text { Electrification of railway, Integrated public } \\
\text { transport, Mass transport collectivization }\end{array}$ \\
\hline & $\begin{array}{l}\text { Efficient freight } \\
\text { management }\end{array}$ & $\begin{array}{l}\text { Repowering, Improved logistics for } \\
\text { multimodal transport, Longer v. heavier } \\
\text { trains }\end{array}$ \\
\hline & $\begin{array}{l}\text { Modal shift in } \\
\text { freight transport }\end{array}$ & $\begin{array}{l}\text { Railway cargo transport, Technologies to } \\
\text { increase use of freight trains }\end{array}$ \\
\hline & Electric vehicles & $\begin{array}{l}\text { Hybrid elecrict vechicles, Battery powered } \\
\text { vehicles }\end{array}$ \\
\hline & $\begin{array}{l}\text { Energy efficient } \\
\text { motors }\end{array}$ & Energy efficient motors and engines \\
\hline & $\begin{array}{l}\text { Non-motorized } \\
\text { transport }\end{array}$ & Bicycles, Cycle lanes \\
\hline & $\begin{array}{l}\text { Vehicle demand } \\
\text { management }\end{array}$ & $\begin{array}{l}\text { Carpool, Four days work and tele-work, Car } \\
\text { sharing, Streamlining procedures }\end{array}$ \\
\hline & $\begin{array}{l}\text { Traffic } \\
\text { management }\end{array}$ & $\begin{array}{l}\text { Energy efficient infrastructures, Transport } \\
\text { management systems, Speed control } \\
\text { of motor drives, Improved traffic signal } \\
\text { systems, Improved road infrastructure, } \\
\text { Electronic road pricing }\end{array}$ \\
\hline
\end{tabular}




\begin{tabular}{|c|c|c|}
\hline $\begin{array}{l}\text { Sector/ } \\
\text { sub-sector }\end{array}$ & Technology class & Within class varieties \\
\hline & $\begin{array}{l}\text { Vehicle and fuel } \\
\text { technologies }\end{array}$ & $\begin{array}{l}\text { Alternative fuels (hydrogen, fuel cells, } \\
\text { blended fuels, CNG and LNG), Promoting } \\
\text { import of fuel efficient vehicles, Vehicle } \\
\text { emission standards, Sustainable private } \\
\text { transport alternatives }\end{array}$ \\
\hline \multirow[t]{6}{*}{ Waste } & Composting & $\begin{array}{l}\text { Composting, Composting in vessel, } \\
\text { Vermiculture, Mushroom cultivation }\end{array}$ \\
\hline & Reuse and recycle & $\begin{array}{l}\text { Recycling of paper and plastic solid waste, } \\
\text { Waste reuse, Using organic waste for flour } \\
\text { production, Heating technology for recyling } \\
\text { tyres }\end{array}$ \\
\hline & $\begin{array}{l}\text { Waste } \\
\text { incineration } \\
\text { for energy } \\
\text { production }\end{array}$ & $\begin{array}{l}\text { Waste to energy, Co-generation from waste } \\
\text { burning }\end{array}$ \\
\hline & Landfill gas & $\begin{array}{l}\text { Methane capture from landfill, Biomass } \\
\text { landfill, Low solid anaerobic digestion }\end{array}$ \\
\hline & $\begin{array}{l}\text { Biological waste } \\
\text { treatment }\end{array}$ & Mechanical-biological treatment (Мвт) \\
\hline & $\begin{array}{l}\text { Wastewater } \\
\text { treatment }\end{array}$ & $\begin{array}{l}\text { Biomass wastewater, Biomethanation, } \\
\text { Wastewater lagooning, Anaerobic digestion }\end{array}$ \\
\hline
\end{tabular}

